\title{
Artificial neural network to predict the natural convection from vertical and inclined arrays of horizontal cylinders
}

\author{
Amin Amiri ${ }^{1 *}$, Alimohammad Karami ${ }^{2}$, Tooraj Yousefi ${ }^{3}$, Mohammad Zanjani ${ }^{4}$ \\ ${ }^{1}$ Kermanshah University of Technology, Department of Chemical Engineering, Kermanshah, Iran \\ ${ }^{2}$ Kermanshah University of Technology, Department of Mechanical Engineering, Kermanshah, Iran \\ ${ }^{3}$ Razi University, Department of Mechanical Engineering, Kermanshah, Iran \\ ${ }^{4}$ Kermanshah University of Technology, Department of Mechanical Engineering, Kermanshah, Iran \\ "Corresponding author: amin.amiri.84@gmail.com
}

\begin{abstract}
The main focus of the present study is to utilize the artificial neural network (ANN) in predicting the natural convection from horizontal isothermal cylinders arranged in vertical and inclined arrays. The effects of the vertical separation spacing to the cylinder diameter ratio $\left(P_{y} / d\right)$, horizontal separation spacing to the cylinder diameter ratio $\left(P_{x} / d\right)$ and Rayleigh number $(R a)$ variation on the average heat transfer from the arrays are considered via this prediction. The training data for optimizing the ANN structure is based on available experimental data. The Levenberg-Marquardt back propagation algorithm is used for ANN training. The proposed ANN is developed using MATLAB functions. For the best ANN structure obtained in this investigation, the mean relative errors of $0.027 \%$ and $0.482 \%$ were reached for the training and test data, respectively. The results show that the predicted values are very close to the experimental ones.
\end{abstract}

Keywords: Natural Convection, Inclined Array, Vertical Array, Modeling, Artificial Neural Network.

\section{INTRODUCTION}

The natural convection heat transfer from the arrays of the parallel horizontal cylinders are encountered in many engineering applications including space heating, the cooling of electronic devices, the heating or cooling of fluids, process plants, oil heating, as well as the cooling of refrigerator condensers. The heat transfer from a cylinder in an array is quite different from a single cylinder ${ }^{1-4}$ due to the interaction of the temperature and flow fields around the neighboring cylinders. This interaction owes to the buoyant plume that is generated by each cylinder and which may impinge on other cylinders. This impingement may either increase or decrease the heat transfer relative to that for a single horizontal cylinder, depending on the position of the cylinders relative to each other. In previous investigations, the cylinders were arranged in a vertical array and in few studies in an inclined array. The first investigation on the natural convection heat transfer from the cylinders in an array was conducted by Eckert and Soehngen ${ }^{5}$. Tokuraet al. ${ }^{6}$ studied the natural convection from vertical arrays of two, three and five cylinders with spacing up to 20 times the cylinder diameter, for Grashof numbers ranging from $4 \cdot 10^{4}$ to $4 \cdot 10^{5}$.The experiments included three horizontal isothermal cylinders of $22.3 \mathrm{~mm}$ diameter in a vertical inline and staggered arrangements. In the case of the vertical array, it was observed that the heat transfer from the lowest cylinder in the array remains the same as a single cylinder, whilst that from the other cylinders, decreases with the elevation in the array. In the staggered arrangement, the natural convection from the bottom cylinder also remains unchanged, but that from the offset middle cylinder improves with respect to the single cylinder. Marsters ${ }^{7}$ conducted an experimental study on the natural convection from a vertical array of three, five and nine cylinders with spacing from 2 to 20 times the cylinder diameter, for Grashof number ranging from 750 to 2000 under the condition of a uniform heat flux. It was found that, even for the closest center-tocenter separation distance, the Nusselt number of the bottom cylinder is substantially identical to that of a single cylinder. In contrast, the Nusselt number of the upper cylinders reduces at close spacing and enhanced at large spacing. Liberman and Gebhart ${ }^{8}$ experimentally investigated the interaction of the heated wires under the condition of a uniform heat flux. The authors used a flat array of 10 wires of $0.127 \mathrm{~mm}$ diameter with six spacing from 37.5 to 225 diameters and four equally orientation angles from $0^{\circ}$ to $90^{\circ}$, for Grashof numbers of the orders of $10^{-1}$. It was reported that there is an optimum spacing at each array angle for a maximum Nusselt number. The highest average Nusselt number occurred at a spacing of 75 times the cylinder diameter for the array at $60^{\circ}$. In another investigation, Rezvantalab et al. ${ }^{9}$ investigated the natural convection from a pair of vertical arrays of the isothermal cylinders experimentally. The aim of the paper was to investigate the effects of the horizontal center-to-center spacing $\left(S_{h} / D\right)$ and the Rayleigh number on the natural convection heat transfer from each vertical array. It was shown that higher values of $S_{h} / D$ lead to an increased average Nusselt number for each individual cylinder in the array. In addition, for small $S_{h} / D$ ratios, flow diverters had a negative effect on the heat transfer. Ashjaee and Yousef $\mathrm{f}^{\mathbf{1 0}}$ studied the natural convection from the vertical and inclined arrays of five isothermal horizontal cylinders. The aim of the paper was to investigate the effects of the cylinder spacing and Rayleigh number on the natural convection heat transfer from vertical and inclined arrays. It was observed that the natural convection from the vertical array has an increasing trend with respect to vertical separation distance. Also in the inclined array, heat transfer increases with increasing the horizontal separation distance and decreases with increasing the vertical separation distance. The current study is mainly focused on the modeling of the natural convection from the horizontal isothermal 
cylinders arranged in vertical and inclined arrays, using an artificial neural network(ANN).The applied experimental data were obtained by Ashjaee and Yousef ${ }^{\mathbf{1 0}}$. The ANN is presently one of the powerful tools widely used for the modeling of various heat transfer processes. Sozen and Arcaklioglu ${ }^{11}$ developed an ANN based model for the Exergy analysis of an ejector-absorption heat transformer. Deng and Hwang ${ }^{12}$ applied neural networks to the solution of forward and inverse heat conduction problems. Zdaniuk et al. ${ }^{13}$ demonstrated the capability of ANN approach in predicting the heat transfer and friction in the helically-finned tubes. Scalabrin and Piazza $^{14}$ presented an ANN model of forced convection heat transfer to the supercritical carbon dioxide inside the tubes. Diaz et al. ${ }^{15}$ applied ANN for the dynamic prediction and control of heat exchangers. Chen et al. ${ }^{16}$ adopted a neuro-based model to predict the heat transfer coefficients of the supercritical carbon dioxide. Hernández et al. ${ }^{17}$ employed the ANN to model the waste energy recovery in a heat transformer in a water purification process. A schematic representation of the problem is shown in Figure 1. The cylinder diameter d, vertical and horizontal center-to-center separation distance $\mathrm{P}_{\mathrm{y}}$ and $\mathrm{P}_{\mathrm{x}}$, the ordinal number of the cylinders $\mathrm{N}_{\mathrm{i}}$, and the height of the array $\mathrm{H}$, are also shown in this figure. a)
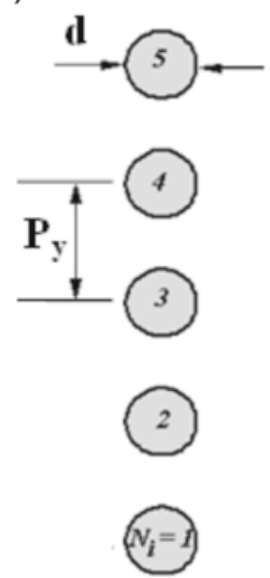

b)

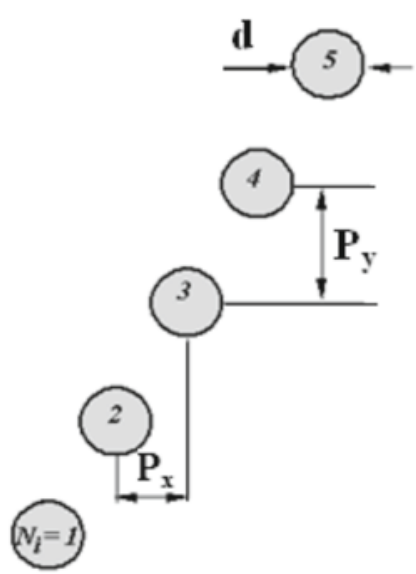

Figure 1. Schematic representation of (a) the vertical array (b) the inclined array

\section{EXPERIMENTAL SETUP}

All the information about the details of the experiment test section can be found elsewhere ${ }^{10}$. A $10 \mathrm{~cm}$ diameter beam Mach-Zehnder Interferometer (MZI) was used in the experimental study. The interferometer consists of a light source, a micro lens, a pinhole, two doublets, two mirrors and two beam splitters (see Figure 2). The used light source was a $30 \mathrm{~mW}$ Helium-Neon laser with $\lambda=632.8 \mathrm{~nm}$. All the interferograms were digitized with a CCD camera (ARTCAM-320P) and the camera was connected to a video recorder through a PC. The local Nusselt numbers at plate surface were obtained using the infinite fringe mode which corresponds directly to the isotherms in the flow field. Further information about MZI is available in literature ${ }^{\mathbf{1 8 , 1 9}}$.

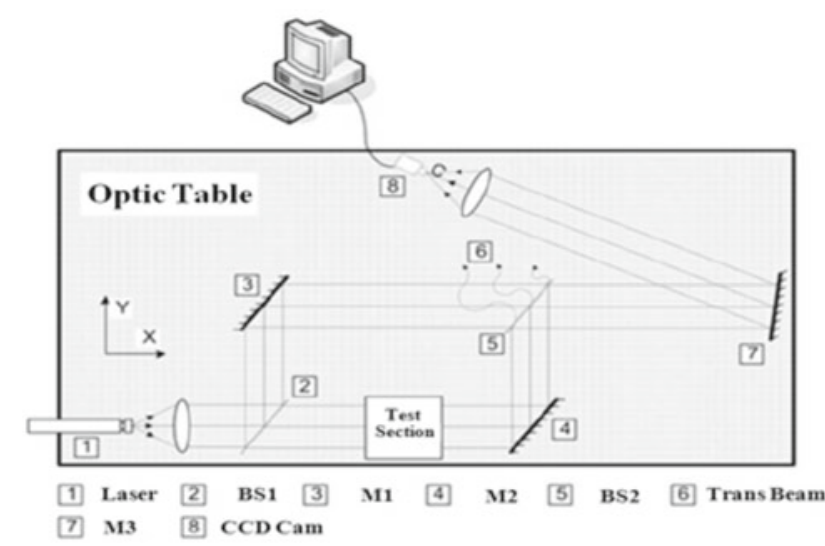

Figure 2. Schematic representation of the Mach-Zehnder Interferometer setup

\section{METHOD OF MODELING}

\section{Computational Intelligence Model}

The artificial neural networks (ANNs) are strong tools for the prediction and simulation in various engineering applications. In this study, the natural convection from the vertical and inclined arrays of horizontal isothermal cylinders, is adopted as a function of three variables, namely the vertical separation spacing to the cylinder diameter ratio $\left(P_{y} / d\right)$, horizontal separation spacing to the cylinder diameter ratio $\left(P_{x} / d\right)$ and Rayleigh number $(R a)$. Therefore, an ANN model as shown in Figure 3 is developed with the vertical spacing ratio $\left(\mathrm{P}_{y} / \mathrm{d}\right)$ ranging from 2 to 5 , horizontal spacing ratio $\left(\mathrm{P}_{x} / \mathrm{d}\right)$ from 0 to 2 and Rayleigh number $(R a)$ from $10^{3}$ to $3 \times 10^{3}$ as inputs and an average Nusselt number of the arrays $\left(\overline{N u}_{a}\right)$ as the desired output.

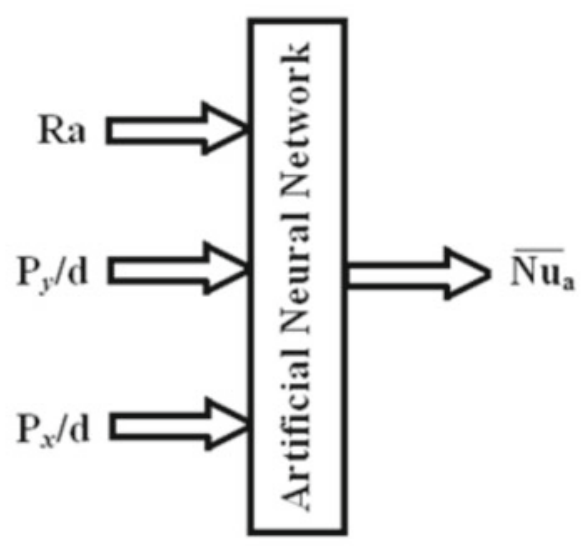

Figure 3. A simplified overview of the proposed ANN model for heat transfer modeling

\section{The ANN Advantages}

The new techniques such as fuzzy $\operatorname{logic}(\mathrm{FL})^{\mathbf{2 0}}$, artificial neural network (ANN) and the adaptive neurofuzzy inference system (ANFIS) consume less time for computation and offer better accuracy as compared to traditional techniques used for the modeling and prediction purposes. In addition, since the data used for developing the ANN, ANFIS ${ }^{21}$ and etc, is based on the training data, therefore, we can test the validity of the proposed models with the test data. Since for developing a correlation, we basically use the total data, therefore it is not possible to carry on with the validity business. It 
is useful mentioning that, among these new techniques, the ANN and ANFIS are widely used for the modeling and prediction purposes. In the current study, the ANN model is preferred due to the following reasons:

a) The speed of training the ANN is more than that of the ANFIS.

b) Two outputs in the ANFIS, require designing two networks, whereas in the ANN case, it is possible to consider more than one output with a single network.

c) Increasing the number of inputs, increases the time of ANFIS training whereas, the ANN training time is affected by the number of inputs but not that much.

d) Adding an extra input to the ANN, requires that an additional neuron with a simple relation (for example a linear or Tansig relation) to be added to the network, but in the ANFIS case by adding a similar input to the network, in fact one membership function having a nonlinear relationship should be added, which in turn increases the computations volume and subsequently, decreases the training speed of the network.

\section{Feed Forward Artificial Neural Networks}

In this study, the feed forward multi-layer perceptron (MLP) network was selected among the main neural network architectures used in engineering. The ANN is constructed as a massive connection model of the simply designed computing unit, called "neuron". Figure 4 illustrates a simple model of the $n$-inputs single-output neuron. All the input signals are summed up as $z$ and the amplitude of the output signal is determined by the nonlinear activation function $f(z)$.

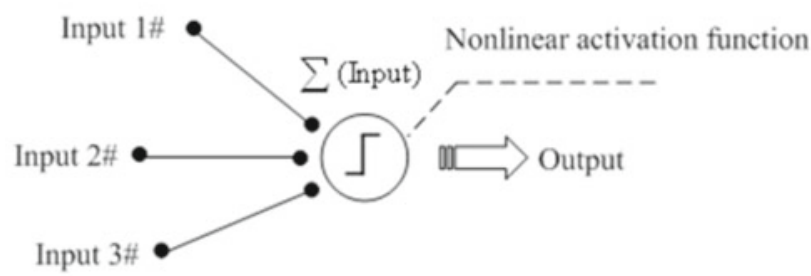

Figure 4. Basic model of the multi-inputs one-output neuron
In this work, the sigmoid function $f(z)$ is used given as follows ${ }^{22}$,

$f(z)=\frac{1-e^{-k z}}{1+e^{-k z}}$

In the limit of $\mathrm{k}=\infty$, as the slope approaches the infinity, $f(z)$ behaves like a threshold function. Here, the sigmoid function is adopted with a moderate slope so that the network can output a continuous range of values from -1 to 1 , which brings the differentiability of the network ${ }^{\mathbf{2 3}}$. Here, a Multilayer Perceptron (MLP) type network is adopted with three layers, which has been used for various applications ${ }^{\mathbf{2 3 - 2 6}}$. The architecture of the perceptron neural network is shown in Figure 5.

For clear notation, the indices $i, j$ and $k$ will be used for the units corresponding to "input", "hidden" and "output" layers, respectively (see Figure 5). Note also that $n_{i}$ and $o_{i}$ are used to represent the input and output to the $i^{\text {th }}$ neuron, respectively. The input-output properties of the neurons in each layer can be simply expressed in a mathematical term $\mathrm{as}^{24}$,

$o_{i}=f\left(n_{i}\right) \quad ; o_{j}=f\left(n_{j}\right) \quad ; \quad o_{k}=f\left(n_{k}\right)$

Whereas the inputs to the neurons are given as,

$n_{i}=($ inputsignaltothe $A N N), n_{j}=\sum_{i=1}^{N_{i}} w_{i j} o_{i}+\theta_{j}$ and

$n_{k}=\sum_{j=1}^{N_{j}} w_{j k} o_{j}+\theta_{k}$

Here, $N_{i}$ and $N_{j}$ represent the numbers of the units belonging to the "input" and "hidden" layers, while $w_{i j}$ denotes the synaptic weight parameter which connects the neurons $i$ and $j$. the threshold parameter (bias) with respect to the neuron $j$ is represented by $\theta_{j}$. We introduced the sigmoid function only in the "hidden" layer to realize smooth and moderate response of the ANN and the linear function for the output layer. This architecture of ANN is a good function approximator ${ }^{\mathbf{2 4}}$. The overall response of the present network is given as, $\left.o_{k}=f_{k}\left[\sum_{j=1}^{N_{j}} w_{j k} f_{j}\left(n_{j}\right)+\theta_{k}\right)\right]$

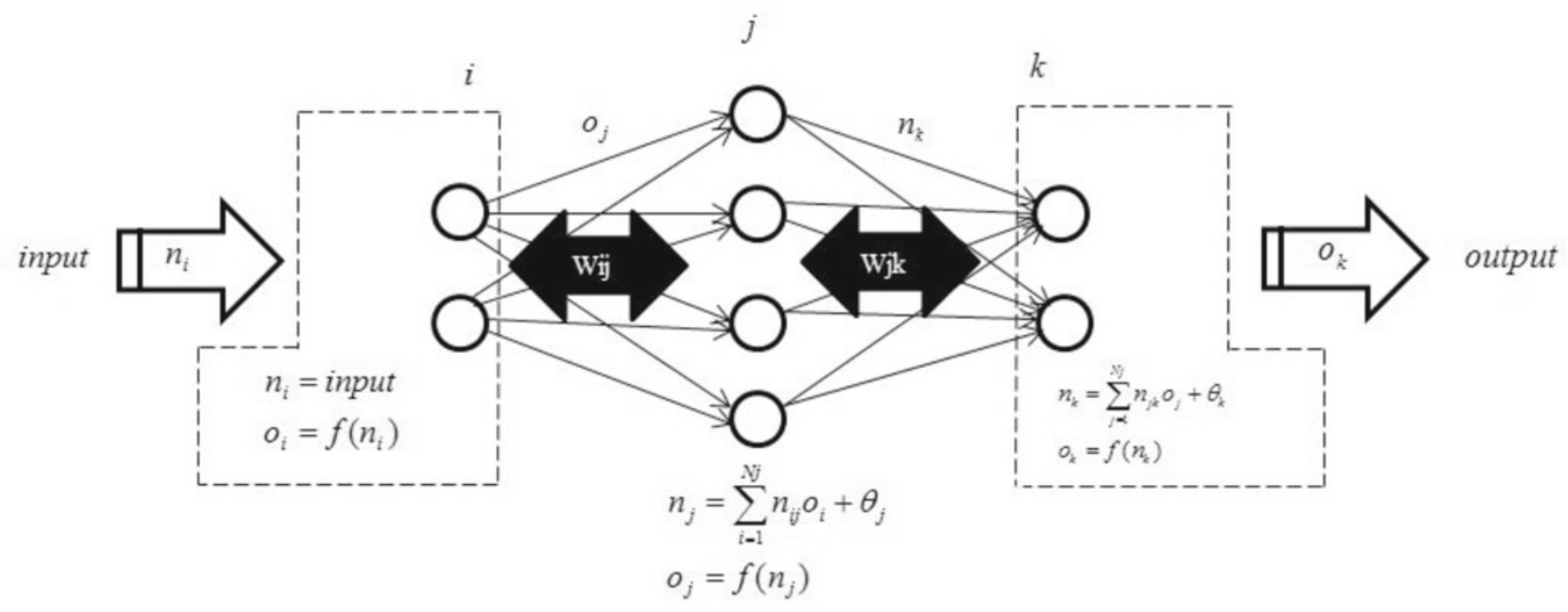

Figure 5. Three layer Multilayer Perceptron consisting of the 'input', 'hidden' and 'output' layers 
Where

$n_{j}=\sum_{i=1}^{N_{i}} w_{i j} o_{i}+\theta_{j}$

ANN training is an optimization process in which an error function is minimized by adjusting the ANN parameters (weights and biases). When an input training pattern is introduced to the ANN, it calculates an output. The output is compared with the real output (experimental data) provided by the user. This difference is used by an optimization technique to train the network. The error function to be minimized in our study is Mean

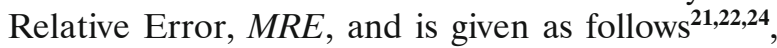

$M R E=\frac{1}{n} \times \sum_{i=1}^{n}\left|\frac{y_{j}-o_{j}}{y_{j}}\right|$

Where, $y_{j}$ is the target data and $o_{j}$ is the output of the neural networks. In our method the target data was the experimental data. The network is trained via the fast convergence gradient-descend back-propagation ${ }^{27}$ method with the momentum term for the nonnegative energy function ${ }^{22,24}$.

\section{MODELING RESULTS AND DISCUSSION}

\section{Applying the ANN Model to the Current Study}

At first, five hundred values for the average Nusselt number of each individual cylinder in the arrays, from the experimental data obtained by Ashjaee and Yousef ${ }^{10}$ are collected. So the average Nusselt number of the vertical array $\overline{N u}_{a v}$ is obtained as the arithmetic mean value of the average Nusselt number of each individual cylinder $\overline{N u}_{i v}$ in the array:

$\overline{N u}_{a v}=\frac{1}{5} \sum_{i=1}^{5} \overline{N u}_{i v}$

For the inclined array a similar process is performed.

The average Nusselt number of the inclined array $\overline{N u}_{a i}$ is obtained as the arithmetic mean value of the average Nusselt number for each individual cylinder in the array $\overline{N u}_{i i}$ :

$\overline{N u}_{a i}=\frac{1}{5} \sum_{i=1}^{5} \overline{N u}_{i i}$

From the equations (6) and (7), one hundred values for the average Nusselt number of the vertical and inclined arrays, is obtained. It is worth mentioning that in order to build up the ANN model, different data sets with various percentages such as $74 \%, 26 \%, 73 \%, 27 \%, 72 \%, 28 \%$, $71 \%, 29 \%, 70 \%, 30 \%$ are used for training and testing as far as the modeling of the process is concerned. Here, in each data set with its associated trained and tested percentage, the number of the hidden layers and also the neurons of each hidden layer are varied and the best results (minimum train and test error) are recorded. In the current study, in each data set, at first, one hidden layer is considered and the number of neurons is varied from 2 to 25 . The best results with the corresponding number of neurons are recorded. If satisfied results are obtained, the structure of the ANN model will consists of one hidden layer with the decisive number of the neurons. If the results are not appropriate, then the second step begins. In this step two hidden layers are considered and the number of neurons is varied in the same range. In this step, again the best corresponding results are recorded. If satisfied results are obtained, the structure of the ANN model will consists of two hidden layers with the decisive number of neurons. Again, if the results are not appropriate, then the third step begins. This process is being iterated so that reasonable results with the low error rate are obtained.

In the present investigation, a network consisting of the percentages of $74 \%$ and $26 \%$ for training and testing, respectively, with the error limits mentioned later, is selected as the best configuration for our network. Moreover, in order to validate the proposed ANN model, sixteen experimental data which are not seen in the training and test data are compared to the predicted one by the ANN model. The best architecture for the proposed neural network is described in table 1 .

Table 1. The optimum architecture and specifications of the proposed ANN model

\begin{tabular}{|c|c|}
\hline Neural network & MLP \\
\hline $\begin{array}{l}\text { Number of the neurons in the } \\
\text { input layer }\end{array}$ & 3 \\
\hline $\begin{array}{l}\text { Number of neurons in the first } \\
\text { hidden layer }\end{array}$ & 5 \\
\hline $\begin{array}{l}\text { Number of the weight parameters } \\
\text { in the first hidden layer }\end{array}$ & 15 \\
\hline $\begin{array}{l}\text { Number of the bias parameters in } \\
\text { the first hidden layer }\end{array}$ & 5 \\
\hline $\begin{array}{l}\text { Number of neurons in the second } \\
\text { hidden layer }\end{array}$ & 11 \\
\hline $\begin{array}{l}\text { Number of the weight parameters } \\
\text { in the second hidden layer }\end{array}$ & 55 \\
\hline $\begin{array}{l}\text { Number of the bias parameters } \\
\text { in the second hidden layer }\end{array}$ & 11 \\
\hline $\begin{array}{l}\text { Number of the neurons in the } \\
\text { output layer }\end{array}$ & 1 \\
\hline $\begin{array}{l}\text { Number of the weight parameters } \\
\text { in the output layer }\end{array}$ & 11 \\
\hline $\begin{array}{l}\text { Number of the bias parameters in } \\
\text { the output layer }\end{array}$ & 1 \\
\hline $\begin{array}{l}\text { Total number of the weight } \\
\text { parameters }\end{array}$ & 81 \\
\hline $\begin{array}{l}\text { Total number of the bias } \\
\text { parameters }\end{array}$ & 17 \\
\hline Number of iterations & 27 \\
\hline Activation function & $\begin{array}{c}\text { Tansig (obtained by setting } \\
\mathrm{k}=1 \text { in equation } 1 \text { ) }\end{array}$ \\
\hline Training function & $\begin{array}{c}\text { Levenberg-Marquardt back } \\
\text { propagation }\end{array}$ \\
\hline
\end{tabular}

\section{Modeling Results}

The training, testing and validation results of the proposed ANN model are shown in Figures 6 to 8 . The comparison between the average Nusselt numbers obtained from the experiments and the predicted one by the ANN model for the vertical array as a function of vertical spacing ratios for some arbitrary Rayleigh numbers is shown in Figure 9. Also, a similar comparison for the inclined array as a function of horizontal spacing ratios for some arbitrary Rayleigh numbers, and vertical spacing ratios is shown in Figure 10. According to these figures and also the results shown in Figures 6 and 7, the maximum errors of the proposed ANN model in predicting the Nusselt number for the training and test data are $0.12 \%$ and $2.44 \%$, respectively. Also the mean relative errors for the training and test data are 


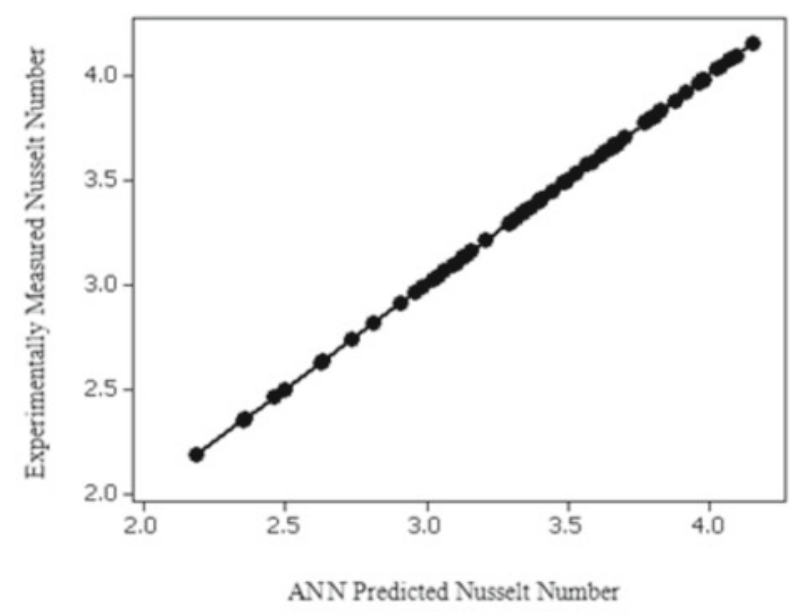

Figure 6. The comparison between the experimental and predicted values of an average Nusselt number using ANN for the training data

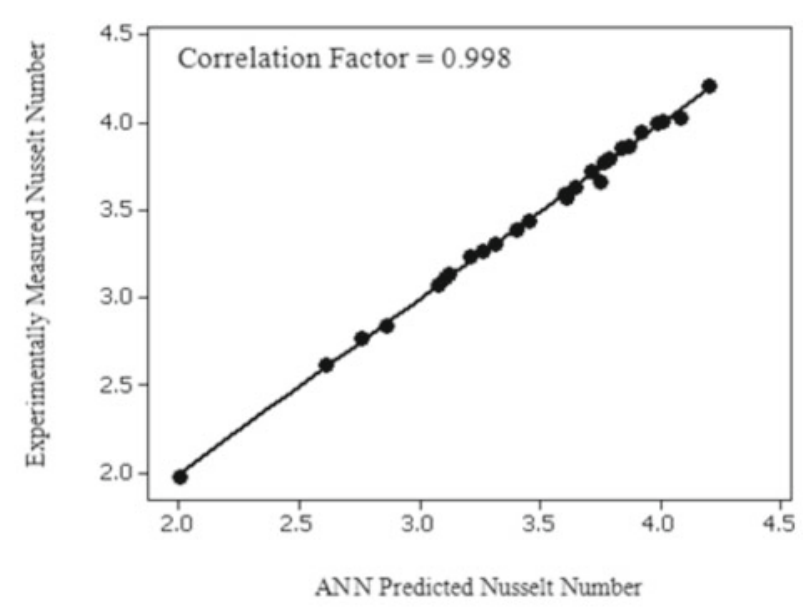

Figure 7. The comparison between the experimental and predicted values of an average Nusselt number using ANN for the testing data

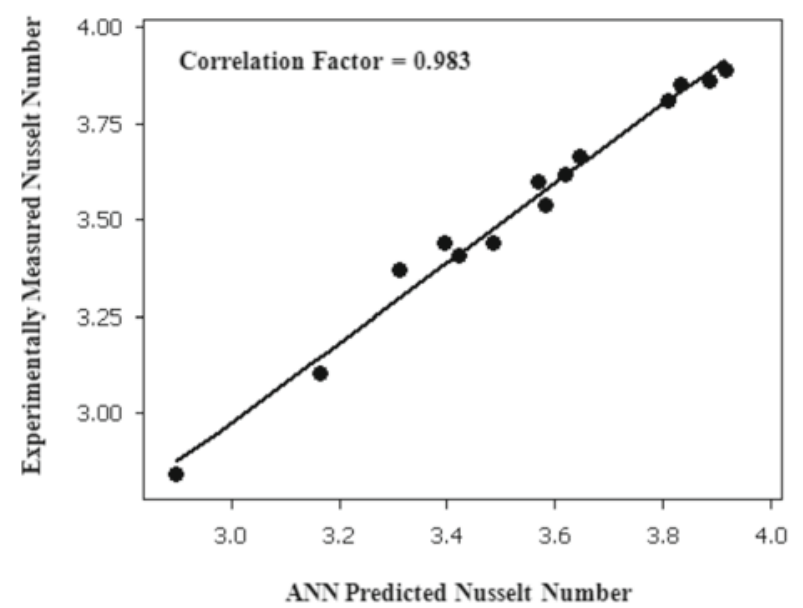

Figure 8. The comparison between the experimental and predicted values of an average Nusselt number using ANN for the validation data

$0.027 \%$ and $0.482 \%$, respectively. In addition, the mean relative error for the validation data is $0.943 \%$. Since, the error values are low, therefore, it can be concluded that there is good consistency between the experimental and predicted results for the training and the test data

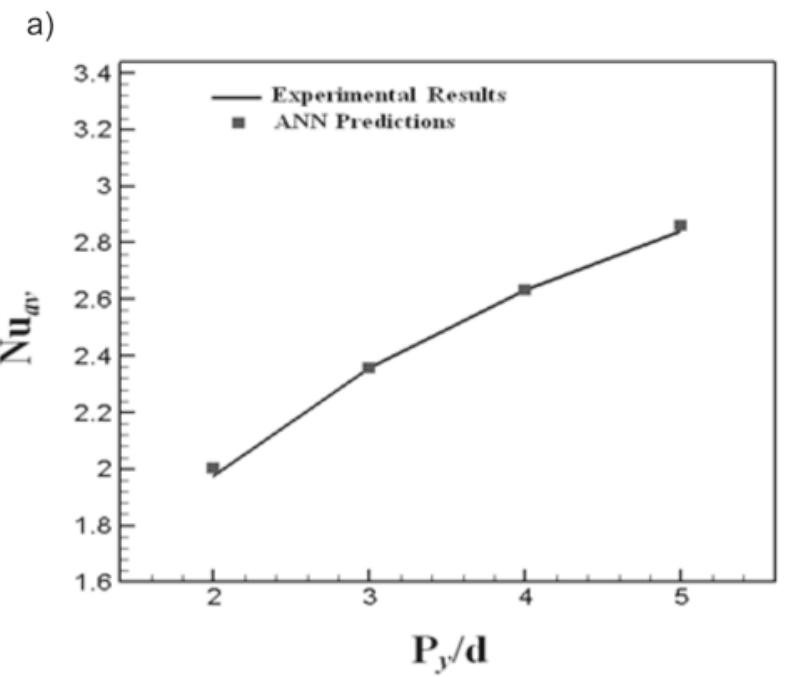

b)

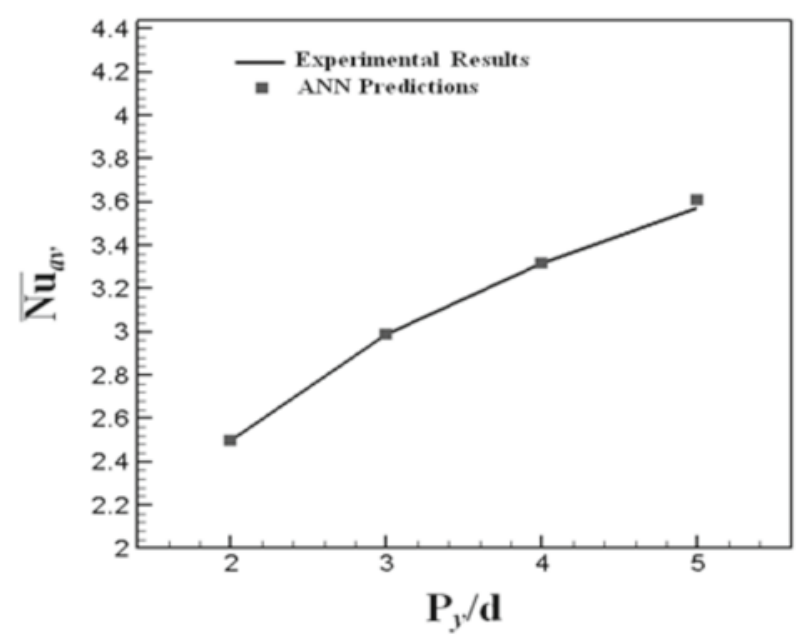

Figure 9. The experimental results obtained by Ashjaee et al. and Yousefi ${ }^{\mathbf{1 0}}$ and the average Nusselt number values of the vertical array predicted using the ANN for a) $\mathrm{Ra}=1.5 \times 10^{3}$ b) $\mathrm{Ra}=2.5 \times 10^{3}$

sets. Hence, the ANN results can be applied to model the experiments precisely. According to Figures 9 and 10 , in the vertical and inclined arrays, heat transfer increases by increasing the Rayleigh number. Figure 9 also indicates that in the vertical array, by increasing the vertical separation spacing, heat transfer increases. In addition, as can be observed in Figure 10, the heat transfer increases by increasing the horizontal spacing ratio and decreases by increasing the vertical spacing ratio. Complete details of the physical interpretation of the effects of the horizontal and vertical separation distance on the heat transfer from both arrays can be found elsewhere ${ }^{10}$.

\section{Comparison of the ANN Model with Traditional Cor- relations}

Ashjaee and Yousef ${ }^{10}$ have presented a correlation for the average Nusselt number of the vertical array as a function of the Rayleigh number and vertical spacing ratio $\left(\mathrm{P}_{\mathrm{y}} / \mathrm{d}\right)$ as follows:

$\overline{N u}_{a v}=R a^{0.235}\left[0.47 \ln \left(P_{y} / d\right)^{0.4}+0.27\right]$

In addition, another correlation has been developed for the inclined array as a function of the Rayleigh number, 
a)

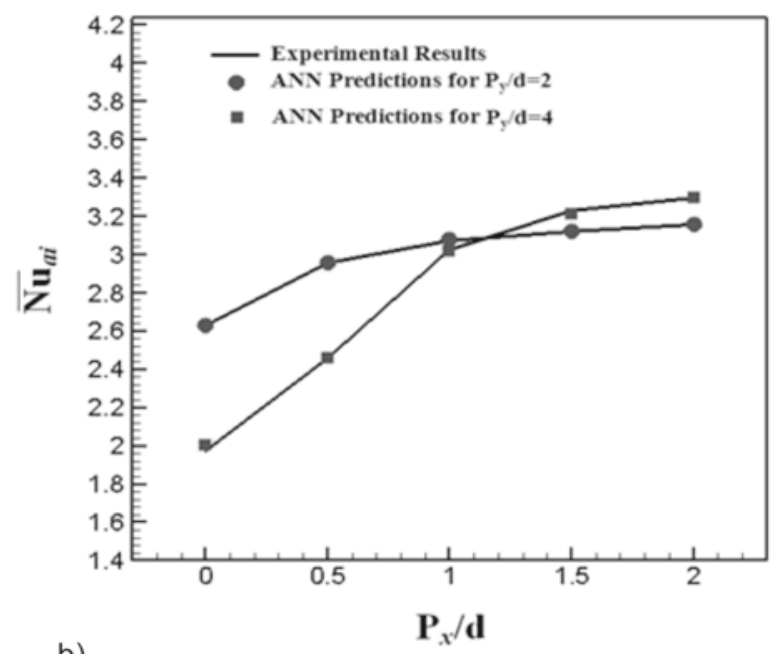

b)

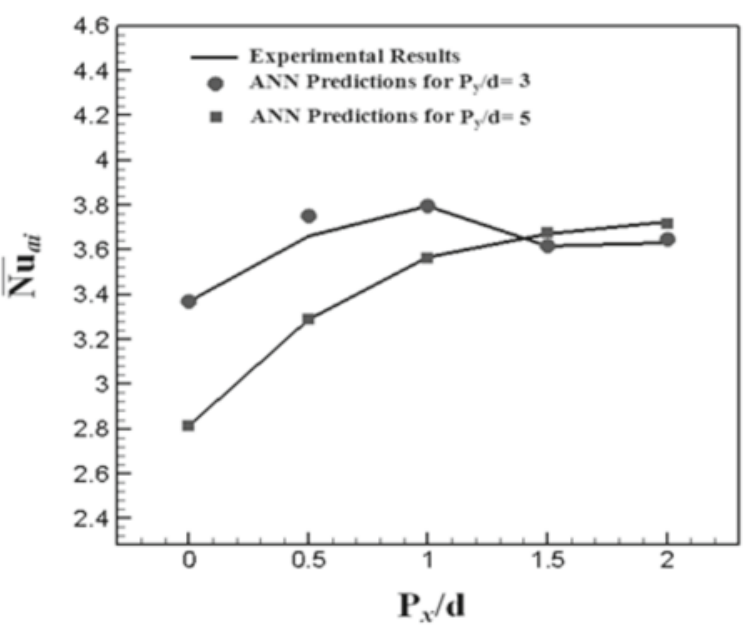

c)

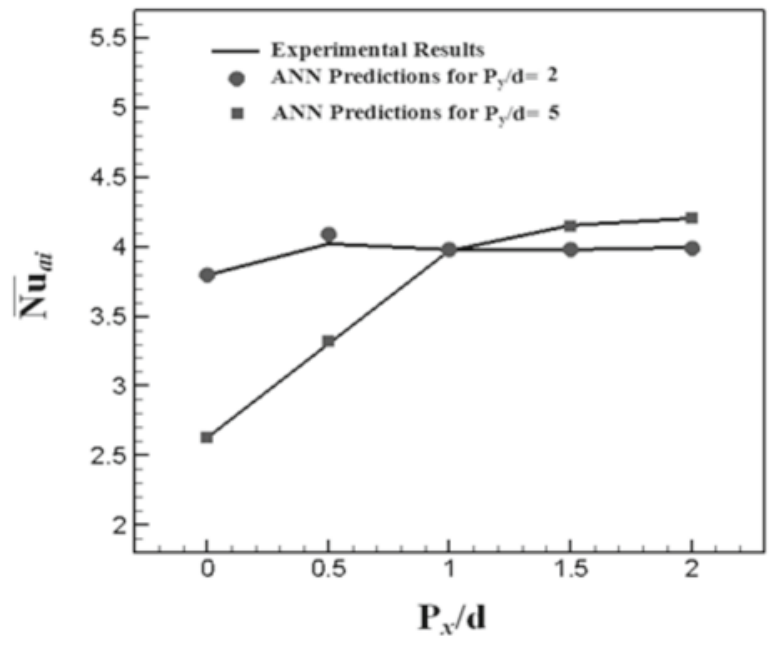

Figure 10. The experimental results obtained by Ashjaee et al. and Yousef ${ }^{10}$ and the average Nusselt number values of the inclined array predicted using the ANN for a) $\mathrm{Ra}=10^{3}$, b) $\left.\mathrm{Ra}=2 \times 10^{3}, \mathrm{c}\right) \mathrm{Ra}=3 \times 10^{3}$

vertical spacing ratio $\left(\mathrm{P}_{y} / \mathrm{d}\right)$ and horizontal spacing ratio $\left(\mathrm{P}_{x} / \mathrm{d}\right)$ as follows:

$\overline{N u}_{a i}=0.572 R a^{0.235}\left[P_{x} / d+0.0256 \cdot e^{\left(0.24 / \eta^{1.07}\right)^{\eta}}\right]$

Where

$\eta=\left(3.115 P_{y} / d\right)^{-0.934}$
The statistical information of the above correlations is presented in table 2. As shown in this table, the Nusselt numbers predicted by the ANN model are closer to those ones predicted by the above correlations.

Table 2. Comparison between the predicted Nusselt numbers using the traditional correlations and ANN model

\begin{tabular}{|c|l|c|}
\hline Predictor type & Array position & MRE $\%$ \\
\hline \multirow{2}{*}{ Traditional Correlations } & Vertical array & 3.49 \\
\cline { 2 - 3 } & Inclined array & 3.98 \\
\hline \multirow{2}{*}{ ANN } & Vertical array & 0.185 \\
\cline { 2 - 3 } & Inclined array & 0.135 \\
\hline
\end{tabular}

\section{CONCLUSIONS}

In this paper, an artificial neural network (ANN) was employed in order to model and predict the natural convection from horizontal isothermal cylinders arranged in vertical and inclined arrays. The comparison between the experimental and predicted values of the proposed ANN model showed that there is excellent consistency between the predicted heat transfer and the experimental results with least error. This means that the proposed ANN model is a reliable flexible mathematical structure for the modeling and prediction of the results due to its high accuracy and therefore, it can be used to simulate the experiments precisely.

\section{Nomenclature}

$d \quad$ diameter of the cylinders, $(\mathrm{m})$

$g \quad$ gravitational acceleration, $\left(\mathrm{m} / \mathrm{s}^{2}\right)$

$l \quad$ length of the cylinder, $(\mathrm{m})$

$N$ number of the cylinders in the array, $N=5$

$N_{i} \quad$ ordinal number of the ith cylinder in the array

$\overline{N u}_{a} \quad$ average Nusselt number of the arrays

$\overline{N u}_{a i} \quad$ average Nusselt number for all the cylinders in the inclined array

$\overline{N u}_{a v} \quad$ average Nusselt number for all the cylinders in the vertical array

$\overline{N u}_{i i} \quad$ average Nusselt number of the $i t h$ cylinder in the inclined array

$p \quad$ pressure, $(\mathrm{Pa})$

$P_{x} \quad$ horizontal center-to-center separation distance, $(m)$

$P_{y} \quad$ vertical center-to-center separation distance, $(m)$

$R a \quad$ Rayleigh number based on the cylinder diameter, $=g \beta\left(T_{w}-T_{\infty}\right) d^{3} / v \alpha$ temperature, $(\mathrm{K})$

Greek symbols

$\alpha \quad$ thermal diffusivity of the air, $\left(\mathrm{m}^{2} / \mathrm{s}\right)$

$\beta \quad$ coefficient of volumetric thermal expansion of the air, $(1 / \mathrm{K})$

$\varepsilon \quad$ fringe shift

$\lambda \quad$ laser wave length $=632.8 \mathrm{~nm}$

$v \quad$ kinematic viscosity of the air, $\left(\mathrm{m}^{2} / \mathrm{s}\right)$

$\varphi \quad$ angle of the flow diverter $=45^{\circ}$

\section{Subscripts}

$w \quad$ referred to the undisturbed air

$\infty \quad$ referred to the cylinder surface

ref referred to a fringe shift $=0$ 


\section{LITERATURE CITED}

1. Kuehn, T.H. \& Goldstein, R.J. (1980).Numerical solution to the Navier-Stokes equations forlaminar natural convection about a horizontal isothermal circular cylinder.Int. J. Heat Mass Transfer. 23(7), 971-979.DOI:10.1016/0017-9310(80)90071-X.

2. Wang, P., Kahawita, R. \& Nguyen, T.H. (1990).Numerical computation of the natural convection flow about a horizontal cylinder using splines. Num. Heat Transfer. 17(2), 191-215. DOI:10.1080/10407789008944739.

3. Saitoh, T., Sajiki, T. \& Maruhara, K. (1993). Bench mark solutions to natural convection heat transfer problem around a horizontal circular cylinder.Int. J. Heat Mass Transfer; 36(5), 1251-1259. DOI: 10.1016/S0017-9310(05)80094-8.

4. Corcione, M. (2005). Correlating equations for free convection heat transfer from horizontal isothermal cylinders set in a vertical array.Int. J. Heat Mass Transfer. 48(17), 3660-3673. DOI:10.1016/j.ijheatmasstransfer.2005.01.010.

5. Eckert, E.R.G. \& Soehngen, E.E. (1948).Studies on heat transfer in laminar free convection with the Zehnder-Mach interferometer.AF Technical Report, 5747, USAF Air Material Command, Wright-Paterson Air Force Base, Ohio.

6. Tokura, I., Saito, H., Kisinami, K. \& Muramoto, K. (1983). An experimental study of free convection heat transfer from a horizontal cylinder in a vertical array set in free space between parallel walls. J. heat Transfer. 105, 102-107.

7. Marsters, G.F. (1972).Array of heated horizontal cylinders in natural convection. Int. J. Heat Mass Transfer. 15(5), 921-933. DOI:10.1016/0017-9310(72)90231-1.

8. Lieberman, J. \& Gebhart, B. (1969).Interaction in natural convection from an array of heated elements, experimental. Int. J. Heat Mass Transfer. 12(11), 1385-1396. DOI: 10.1016/00179310(69)90023-4.

9. Rezvantalab, H., Ghazian, O., Yousefi, T. \& Ashjaee, M. (2011). Effect of flow diverters on free convection heat transfer from a pair of vertical arrays of isothermal cylinders. Experimental Thermal and Fluid Science. 35(7), 1398-1408. DOI: 10.1016/j.expthermflusci.2011.05.008.

10. Ashjaee, M. \& Yousefi, T. (2007). Experimental Study of Free Convection Heat Transfer from Horizontal Isothermal Cylinders Arranged in Vertical and Inclined Arrays. J. Heat Transfer Engineering.28(5),460-471.DOI: 10.1080/01457630601165822.

11. Sozen, A. \& Arcaklioglu, E. (2007).Exergy analysis of an ejector-absorption heat transformer using artificial neural network approach. Appl. Therm. Eng. 27(2-3), 481-491.DOI:10.1016/j.applthermaleng.2006.06.012.

12. Deng, S. \& Hwang, Y. (2006).Applying neural networks to the solution of forward and inverse heat conduction problems.Int. J. of Heat and Mass Transfer.49(25-26), 4732-4750. DOI: 10.1016/j.ijheatmasstransfer.2006.06.009.

13. Zdaniuk, G.J., Chamra, L.M. \& Walters, D.K. (2007).Correlating heat transfer and friction in helically-finned tubes using artificial neural networks.Int. J. of Heat and Mass Transfer 50(2324), 4713-4723. DOI: 10.1016/j.ijheatmasstransfer.2007.03.043.

14. Scalabrin, G. \& Piazza, L. (2003).Analysis of forced convection heat transfer to supercritical carbon dioxide inside tubes using neural networks. Int. J. of Heat and Mass Transfer 46(7), 1139-1154.DOI: 10.1016/S0017-9310(02)00382-4.

15. Diaz, G., Sen, M., Yang, K.T. \& McClain, R.L. (2001). Dynamic prediction and control of heat exchangers using artificial neural networks. Int. J. of Heat and Mass Transfer.44(9), 1671-1679. DOI: 10.1016/S0017-9310(00)00228-3.

16. Chen, J., Wang, Kuan-Po. \& Liang, M-Tsai. (2005).Predictions of heat transfer coefficients of supercritical carbon dioxide using the overlapped type of local neural network. Int. J. of Heat and Mass Transfer.48(12), 2483-2492. DOI:10.1016/j. ijheatmasstransfer.2004.12.040.

17. Hernández, J.A., Romero, R.J., Juárez, D., Escobar, R.F. \& Siqueiros, J. (2009). A neural network approach and thermodynamic model of waste energy recovery in a heat transformer in a water purification process. Desalination. 243(13), 273-285. DOI: 10.1016/j.desal.2008.05.015.

18. Hauf, W. \& Grigull, U. (1970). Optical methods in heat transfer. Advances in Heat Transfer. 6, Academic Press, New York, 133-366.

19. Eckert, E.E.R.G. \& Goldstein, R.J. (1972). Measurements in Heat Transfer.second edition, McGraw-Hill, New York, 241-293.

20. Karami, A., Rezaei, E., Shahhosseni, M. \& Aghakhani, M. (2012). Fuzzy logic to predict the heat transfer in an air cooler equipped with different tube inserts. Int. J. of Therm. Sci. 53, 141-147. DOI: 10.1016/j.ijthermalsci.2011.10.016.

21. Rezaei, E., Karami, A., Yousefi, T. \& Mahmoudinezhad, S. (2012).Modeling the free convection heat transfer in a partitioned cavity using ANFIS, Int. Communications in Heat and Mass Transfer. 39(3), 470-475.DOI: 10.1016/j.icheatmasstransfer.2011.12.006.

22. Minai, A.A. \& Williams R.D. (1990). Acceleration of back propagation through learning rate and momentum adaptation. International joint conference on neural networks; 1, 676-9.

23. Neural Computing, (1996). A technology handbook for professional II/ PLUS and neural works explorer,. Pittsburgh: Neural Ware Inc, Technical Publications Group.

24. Haykin, S.(1994). Neural networks: a comprehensive foundation, New York: Macmillan College Publishing Company; ISBN 0-02352761-7.

25. Hammouda, HB,.Mhiri, M., Gafsi, Z., Besbes, K. (2008). Neural-based models ofsemiconductor devices for HSPICE Simulation. Am. J. Appl.Sci. 5(4), 385-391.DOI: 10.3844/ ajassp.2008.385.391.

26. Shirvany, Y., Hayati, M., Moradian, R. (2008).Numerical solution of the nonlinear Schrodinger equationbyfeedforward neural networks.Communications in Nonlinear Science and Numerical Simulation. 13(10), 2132-2145. DOI: 10.1016/j. cnsns.2007.04.024.

27. Gallant, AR. \& White, H. (1992).On learning the derivatives of an unknown mappingwith multilayer feed forward networks. Elsevier Science.5, 129-38. 